\title{
Construction and comprehensive analysis of a ceRNA network to reveal potential prognostic biomarkers for lung adenocarcinoma
}

Lei Gao ${ }^{1+}$ and Ling Zhang ${ }^{2^{*}+}$

\begin{abstract}
Background: More and more studies have proven that circular RNAs (circRNAs) play vital roles in cancer development via sponging miRNAs. However, the expression pattern of competing endogenous RNA (ceRNA) in lung adenocarcinoma (LUAD) remains largely unclear. The current study explored functional roles and the regulatory mechanisms of circRNA as ceRNAs in LUAD and their potential impact on LUAD patient prognosis.

Methods: In this study, we systematically screened differential expression circRNAs (DEcircRNAs), miRNAs (DEmiRNAs) and mRNAs (DEGs) associated with LUAD. Then, DEcircRNAs, DEmiRNAs and DEGs were selected to construct a circRNA-miRNA-mRNA prognosis-related regulatory network based on interaction information from the ENCORI database. Subsequently, the gene ontology (GO) and Kyoto Encyclopedia of Genes and Genomes (KEGG) pathway enrichment analysis were performed on the genes in the network to predict the potential underlying mechanisms and functions of circRNAs in LUAD. In addition, Kaplan-Meier survival analysis was performed to evaluate clinical outcomes of LUAD patients, and drug sensitivity analysis was used to screen potential biomarkers for drug treatment of patients with LUAD.

Results: As a result, 10 circRNAs were aberrantly expressed in LUAD tissues. The ceRNA network was built, which included 3 DEcircRNAs, 6 DEmiRNAs and 157 DEGs. The DEGs in the ceRNA network of hsa_circ_0049271 enriched in biological processes of cell proliferation and the Jak-STAT signaling pathway. We also detected 7 mRNAs in the ceRNA network of hsa_circ_0049271 that were significantly associated with the overall survival of LUAD patients $(P<0.05)$. Importantly, four genes (PDGFB, CCND2, CTF1, IL7R) identified were strongly associated with STAT3 activation and drugs sensitivity in GDSC.
\end{abstract}

\footnotetext{
* Correspondence: zhangling86061@163.com; jasonxu@xmu.edu.cn

†Lei Gao and Ling Zhang contributed equally to this work.

${ }^{2}$ Department of Critical Care Medicine, The First Affiliated Hospital of Anhui

Medical University, 218 Jixi Road, Shushan District, Hefei 230022, Anhui

Province, China

Full list of author information is available at the end of the article
}

(c) The Author(s). 2021 Open Access This article is licensed under a Creative Commons Attribution 4.0 International License, which permits use, sharing, adaptation, distribution and reproduction in any medium or format, as long as you give appropriate credit to the original author(s) and the source, provide a link to the Creative Commons licence, and indicate if changes were made. The images or other third party material in this article are included in the article's Creative Commons licence, unless indicated otherwise in a credit line to the material. If material is not included in the article's Creative Commons licence and your intended use is not permitted by statutory regulation or exceeds the permitted use, you will need to obtain permission directly from the copyright holder. To view a copy of this licence, visit http://creativecommons.org/licenses/by/4.0/. The Creative Commons Public Domain Dedication waiver (http://creativecommons.org/publicdomain/zero/1.0/) applies to the data made available in this article, unless otherwise stated in a credit line to the data. 
Conclusions: In summary, a ceRNA network of hsa_circ_0049271 was successfully constructed, which including one circRNA, two miRNAs, and seven mRNAs. Seven mRNAs (PDGFB, TNFRSF19, CCND2, CTF1, IL11RA, IL7R and MAOA) were remarkably associated with the prognosis of LUAD patients. Among seven mRNA species, four genes (PDGFB, CCND2, CTF1, and IL7R) could be considered as drug targets in LUAD. Our research will provide new insights into the prognosis-related ceRNA network in LUAD.

Keywords: Circular RNA, Lung adenocarcinoma, Microarray expression, Competing endogenous RNA, Overall survival, Prognostic biomarker, Drug sensitivity

\section{Background}

Lung cancer is the most common cause of cancerrelated mortality worldwide. Lung adenocarcinoma (LUAD) is the leading subtype of all lung cancers, accounting for about 40 to $50 \%$ of all lung cancer cases [1]. Despite improvements in diagnosis and the considerable research into cancer therapy, the average 5 years survival rate of LUAD patients is still less than 20\% [2], because most patients are usually diagnosed at late stages and have little opportunity for effective treatment [3]. Therefore, identifying a novel accurate and sensitive biomarker for the early diagnosis of LUAD patients is urgently needed.

Circular RNAs are a new type of endogenous noncoding RNA, derived from precursor mRNA back-splicing. Circular RNAs (circRNAs) have a circular covalently closed structure without $5^{\prime}$ caps and $3^{\prime}$ poly tails and higher tolerance to exonuclease digestion [4]. Compared to linear counterparts, circRNAs are highly stable and conservative [5], and which can be found in exosomes, plasma [6] and even in urine, and could be a new noninvasive biomarker [7]. Over the past few decades, these transcripts have been considered a by-product of RNA splicing and have long been ignored [8]. With the advent of high throughput sequencing technologies and development of computational technologies, thousands of circRNAs are detected in various organisms, particularly tumors [9]. Growing evidence demonstrated that circRNAs were involved in the initiation and development of cancer [10]. Recent studies proved that some circRNAs can act as a molecular sponge of miRNAs to regulate gene expression. For instance, up-regulating circRNA-MYLK can promote epithelial-mesenchymal transition in bladder cancer [11], CircHIPK3 acts as a miR-7 sponge to promote colorectal cancer growth and metastasis [12], circNRIP1 sponges miR-149-5p to promote gastric cancer progression [13], pro-metastasis process of circANKS1B in breast cancer [14]. Similarly, differentially expressed circRNAs were also identified in LUAD, such as hsa_circ_0020732 [15] and hsa_circ_ 0128332 [16] enhanced LUAD metastasis, hsa_circ 0006427 [17] suppressed LUAD progression, hsa_circ 0012673 [18] could promote LUAD proliferation, indicating that circRNAs play important roles in LUAD pathogenesis. Without a doubt, circRNAs could act as ceRNA by sponging miRNA to relieve the repression of miRNAs for their targets $[19,20]$. Therefore, circRNAs may be potential biomarkers or therapeutic targets.

In the present study, we have downloaded microarray datasets, GSE101684 [21], GSE101586 [22] and GSE29249 [23] from Gene Expression Omnibus (GEO, https://www.ncbi.nlm.nih.gov/geo/), the expression profiles of miRNAs and mRNAs in Cancer Genome Atlas (TCGA)-LUAD dataset. The expression levels of circRNA, miRNA and mRNA were comprehensively analyzed in LUAD. In order to identify the potential biological functions of the circRNA, differential expression of three kinds of RNA were screened to construct circRNA-miRNA-mRNA ceRNA network. Next, functional enrichment analysis was performed to reveal biological process and Jak-STAT signaling pathway of the ceRNA network in LUAD. The survival analysis demonstrated that seven mRNAs (PDGFB, TNFRSF19, CCND2, CTF1, IL11RA, IL7R and MAOA) regulated by circRNA (hsa_circ_0049271) were prognostic biomarkers in LUAD. Finally, there are some potential drugs which are specifically associated with mRNAs, indicating that effects of drugs in LUAD. Our findings suggested that the dysregulation of circRNAs in LUAD could be a novel diagnosis and treatment biomarkers (Fig. 1a).

\section{Methods \\ Microarray data and RNA-Seq data}

The circRNA and mRNA expression profiles in LUAD were obtained from GEO database. GSE101684 from the platform GPL21825, 074301 Arraystar Human circRNA microarray V2, which includes tumor samples and paired adjacent normal tissues from 4 patients. GSE101586 from GPL19978, Agilent-069978 Arraystar Human circRNA microarray V1, which provides five LUAD and five non-tumor adjacent lung tissue samples. GSE29249 from the platform GPL10558, Illumina HumanHT-12 V4.0 expression beadchip, which consists of six paired NSCLC cancer tissue and the adjacent normal tissue. Series Matrix File(s) and platform information were downloaded by 'GEOquery' $\mathrm{R}$ package. The expression levels of miRNAs and mRNAs in TCGALUAD dataset were also downloaded from the Cancer 

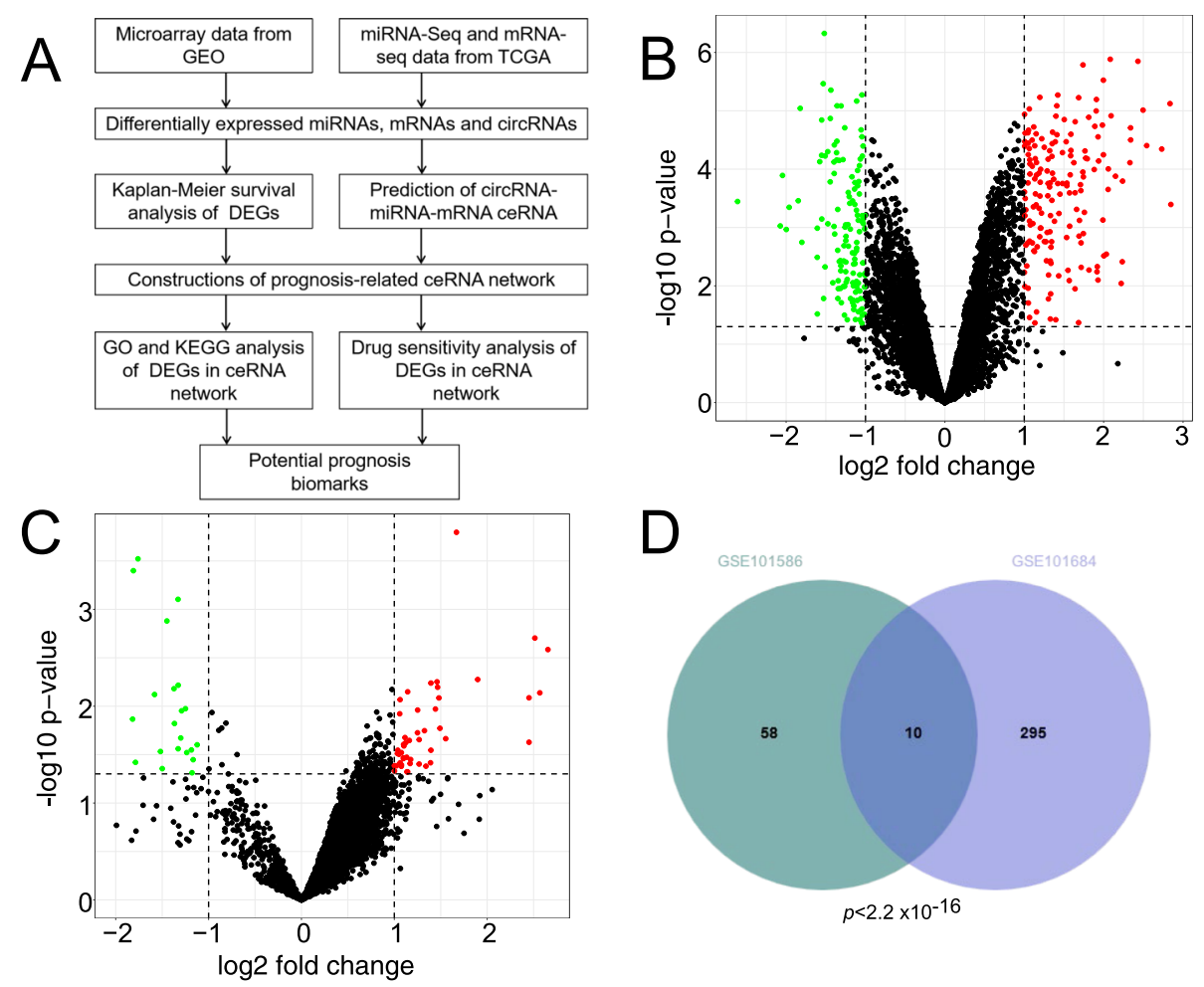

D

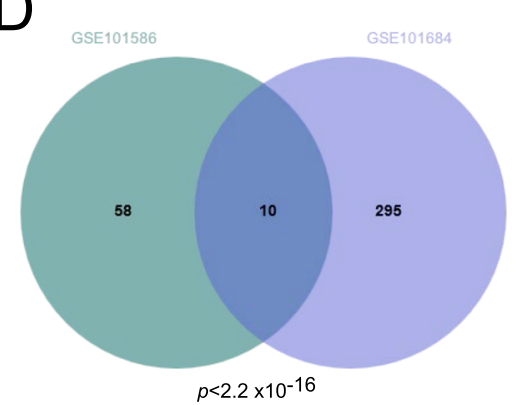

Fig. 1 Volcano plot of differentially expressed circRNAs in LUAD. A Schematic plot for the identification and analysis of prognostic circRNAs in the ceRNA network (B) Differentially expressed circRNAs in GSE101684 dataset. C Differentially expressed circRNAs in GSE101586 dataset. D The overlap of circRNA transcripts detected in GSE101684 dataset and GSE101586 dataset was significant

Browser (https://xena.ucsc.edu/welcome-to-ucsc-xena/) [24]. The detailed dataset information is described in (Table 1).

\section{Identification of differentially expressed transcript in LUAD samples}

Log2 transformation was performed for each circRNA, miRNA and mRNA expression data. The probe name of circRNA, miRNA and mRNA were converted into circBase's ID, mirBase's ID and gene symbol respectively $[25,26]$. The R package 'limma' in Bioconductor [27] or t test was used to implement circRNA, miRNA and mRNA differential expression. circRNA, miRNA and mRNA with the threshold set at a $P$-value $<0.05$ and $\mid \log 2$-fold change $(\mathrm{FC}) \mid>1$ were considered as differentially expressed circRNA (DEcirRNAs), miRNA
(DEmiRNAs) or mRNA (DEGs). A hierarchical cluster heatmap based on Euclidean distance was generated using the 'pheatmap' $\mathrm{R}$ package.

\section{Network analysis of ceRNA and function annotation}

CircRNAs can function as miRNA sponges to bind to miRNAs and modulate downstream target genes of miRNAs. Based on the ENCORI database (http://starbase. sysu.edu.cn/), miRNA-circRNA pair and miRNA-mRNA pair information were obtained. To improve the ceRNA network reliability, DEcirRNAs, DEmiRNAs and DEGs were chosen to construct the ceRNA network. Additionally, miRNAs are negative regulators of gene expression, therefore, expression of the miRNA and mRNA in a ceRNA network should be negatively correlated, and expression of the circRNA and mRNA in a ceRNA nework

Table 1 Details for GEO and TCGA lung adenocarcinoma cancer data

\begin{tabular}{|c|c|c|c|c|c|}
\hline Reference & Dataset & Platform & Normal & Tumor & RNA type \\
\hline Zhao et al (2017) [21] & GSE101684 & GPL21825 & 4 & 4 & circular RNA \\
\hline Chen et al (2019) [22] & GSE101586 & GPL19978 & 5 & 5 & circular RNA \\
\hline Ma L et al (2011) [23] & GSE29249 & GPL10558 & 6 & 6 & mRNA \\
\hline Goldman et al (2020) [24] & mRNA_HiSeq & & 45 & 450 & mRNA \\
\hline Goldman et al (2020) [24] & miRNA_HiSeq & & 45 & 450 & miRNA \\
\hline
\end{tabular}


should be positively correlated [28]. The ceRNA network was visualized using the 'ggalluvial' $\mathrm{R}$ package. To obtain a more comprehensive understanding of the potential mechanism and biological function of the ceRNA network, we implemented Gene Ontology (GO) and Kyoto Genome Encyclopedia (KEGG) [29] pathway analyses using the GESA online analysis database for DEGs in the ceRNA network [30]. The significant enrichment GO term and KEGG pathway were selected at a $P$ value < 0.05 .

\section{Survival analysis}

The survival information of LUAD samples were downloaded from the Cancer Browser. After combining the overall survival of patients and the expression of DEGs in the ceRNA network, the 'survival' and 'survminer' $\mathrm{R}$ package were used to implement a survival analysis and draw Kaplan-Meier plot. The patients were classified into two groups (high vs. low) using median expression levels of DEGs. Log-rank $P<0.05$ was considered significant.

\section{Drug-DEGs associations across cancer cell lines}

GSCALite is a web-based analysis platform for gene set cancer analysis [31]. To investigate the effects of drug sensitivity on DEGs expression, GSCALite web tool was used to perform drug analysis. DEGs as input gene identifiers were submitted online for gene set analysis. Then the Spearman rank correlations were calculated between DEGs expression levels and IC50 values of drugs sensitivity across cancer cell lines in GDSC $[32,33]$. Correlations with false discovery rate less than 0.05 were considered as significant pairs.

\section{Results}

Identification of differentially expressed circRNAs in LUAD Microarray GSE101684 and GSE101586 dataset were reanalyzed to identify differentially expressed circRNAs in LUAD and matched adjacent normal tissues. There were 305 DEcircRNAs (168 upregulated DEcircRNAs and 137 downregulated DEcircRNAs) from GSE101684 dataset, which were shown in (Fig. 1b and Table S1). A total of 68 DEcircRNAs (47 upregulated DEcircRNAs and 21 downregulated DEcircRNAs) were obtained from GSE101586 dataset, which were shown in (Fig. 1c and Table S2). The cluster heatmaps of the top 10 DEcircRNAs for both datasets were shown in (Figure S1A and Figure S1B). The overlapped DEcircRNAs found in GSE101684 and GSE101586 dataset were significant (Fisher Exact Test $p<2.2 \times 10-16$ ) (Fig. 1d). In order to find key circRNAs that are dysregulated in LUAD, we choose 10 overlapped DEcircRNAs for further analysis. Finally, five upregulated (hsa_circ_0000519, hsa_circ 0003528, hsa_circ_0008274, hsa_circ_0072088 and hsa circ_0082564) and five downregulated circRNAs (hsa circ_0000662, hsa_circ_0003162, hsa_circ_0029426, hsa circ_0043256 and hsa_circ_0049271) were identified as candidate circRNAs.

\section{Identification of differentially expressed genes in LUAD}

When GSE29249 dataset was screened by the 'limma' R package, 1742 differentially expressed genes (DEGs) were obtained. Among them, 932 upregulated genes and 810 downregulated genes were identified (Figure S2A and Table S3). In addition, 4222 DEGs were obtained from TCGA-LUAD, which included 1729 upregulated genes and 2493 downregulated genes (Figure S2B and Table S4). The cluster heatmaps of the top 50 DEGs were shown in (Fig. 2a). The overlapped DEGs in GSE29249 and TCGA-LUAD dataset were significant (Fisher Exact Test $p<2.2 \times 10-16$ ) (Figure S2C). Moreover, in order to investigate their prognostic value of DEGs regulated by circRNAs in LUAD, clinical information from TCGA-LUAD dataset were used.

Next, we analyzed the expression levels of circRNAs' host genes in TCGA-LUAD, only $7(0.17 \%)$ host genes associated with DEcircRNAs from GSE101586 dataset and $42(0.99 \%)$ host genes associated with DEcircRNAs from GSE101684 dataset were found (Fig. 2b). These results indirectly suggested that expression levels of the circRNAs are not a reliable mediator of the corresponding abundance of host genes. Previous studies have demonstrated the average abundance of the host genes only weakly correlated with the average abundance of their associated circRNAs [4]. Another mechanism, that circRNAs can regulate genes in trans by absorbing microRNA, rather than induces host genes in cis. In other words, miRNA might play an important role in regulating ceRNA events in LUAD. Therefore, differentially expressed miRNAs (Fig. 2c and Table S5), circRNAs and genes were chosen to construct the network of ceRNA.

\section{Construction of a ceRNA network in LUAD}

In order to further understand the effect of circRNAs on mRNA regulated by combination with miRNAs in LUAD, we established the ceRNA network. The ceRNA network consisted of $3(30 \%)$ circRNAs of 10 DEcircRNAs, 6 (4.08\%) miRNAs of 147 DEmiRNAs and 157 (3.72\%) unique mRNA of 4222 DEGs, which were visualized with the ggalluvial $\mathrm{R}$ package (Fig. 3a). Moreover, we observed 3 DEmiRNAs were bound to hsa_circ 0049271 (from GSE101684, $\log _{2} \mathrm{FC}=-1.13, P<1.70 \times$ $10^{-4}$, Table S1 and Table S6), which mediated 127 unique DEGs. In addition, we also found hsa_circ 0029426 (from GSE101684, $\log _{2} \mathrm{FC}=-1.96, P<4.06 \times$ $10^{-3}$, Table S1, Table S6 and Figure S3A) and hsa_circ 0043256 (from GSE101684, $\log _{2} \mathrm{FC}=-1.39, P<4.90 \times$ $10^{-4}$, Table S1, Table S6 and Figure S3B) sponging at 


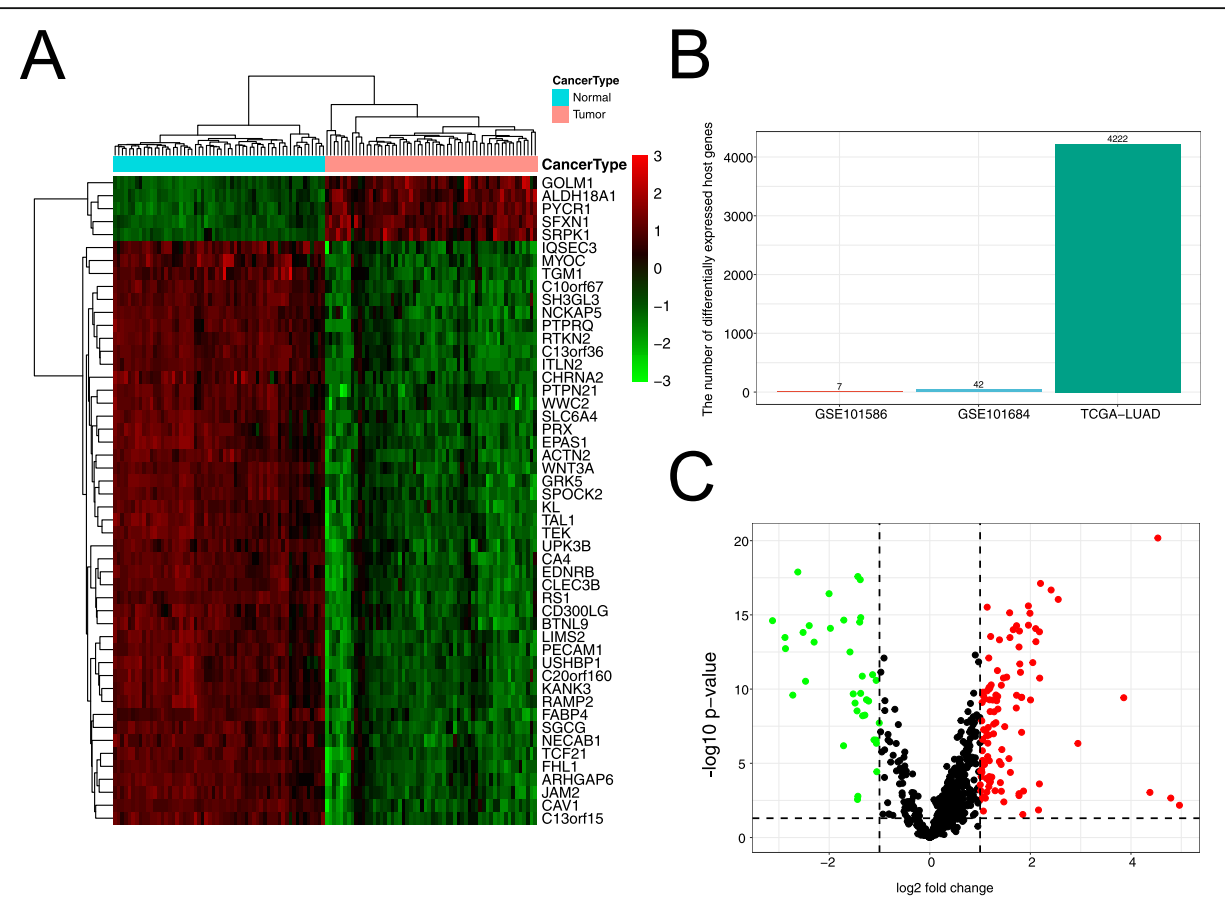

Fig. 2 RNA expression profiles in LUAD and matched normal tissues. A Heatmap of the differential expression of the top 50 DEGs in TCGA-LUAD dataset. B The number of differentially expressed host genes associated with DEcircRNAs. C Differentially expressed miRNAs in TCGA-LUAD dataset

least 1 different DEmiRNA to regulate 26 and 17 DEGs respectively. Collectively, these data suggested that hsa circ_0049271, hsa_circ_0029426 and hsa_circ_0043256 may play important roles in LUAD.

\section{Functional enrichment analysis of DEGs in the ceRNA network}

Gene ontology analysis with 127 DEGs regulated by hsa circ_0049271 in the ceRNA network was conducted. The top 10 significant GO term results showed that hsa circ_0049271 were divided into 2 groups, which consisted in cellular component and biological process groups (Table S7). These GO term results showed that hsa_circ_0049271 were mainly involved in biological processes of the progression of the circulatory system over time and any process that modulates the frequency, rate or extent of cell proliferation (Fig. $3 \mathrm{~b}$ and Table S7). To identify the biological pathways related to hsa_circ 0049271, we conducted GSEA based on 127 DEGs. Then 3 pathways were identified, which were Cytokinecytokine receptor interaction, Jak-STAT signaling pathway and Tyrosine metabolism (Fig. 3c and Table S8).

We also analyzed expression profiles of their target genes in the significant KEGG pathway, and there were 7 prognostic risk target genes of hsa_circ_0049271 in the ceRNA network (Fig. 4). Finally, one circRNA (hsa circ_0049271), two miRNAs (hsa-miR-3127-5p and hsamiR-1306-5p), and seven mRNAs (PDGFB, TNFRSF19,
CCND2, CTF1, IL11RA, IL7R and MAOA) were selected. The hsa_circ_0049271 was identified to be low expressed in LUAD, the decreased expression of hsa circ 0049271, and the decreased expression of TNFR SF19, CCND2, CTF1, IL11RA, IL7R and MAOA in LUAD patients had a short OS period, which may be suppressor genes in LUAD patients. However, the decreased expression of PDGFB demonstrated a good prognosis and potentially could be a promoted cancer factor (Fig. 4 and Fig. 5). In addition, 15 DEGs regulated by other two circRNAs (hsa_circ_0029426 and hsa_circ_ 0043256) were significantly associated with clinical outcomes of patients with LUAD (Figure S4). These results showed that these genes were promising potential diagnostic biomarkers for LUAD patients.

\section{Contribution of DEGs as a predictor of anticancer drug response}

To identify drug-related DEGs in LUAD, we thus explored the website GSCALite, the above seven target genes were selected for drug sensitivity analysis to find some potential drugs. The drug-DEGs associations results were obtained (Fig. 6). Analysis of these drugDEGs associations, we observed that IL11RA was associated with one drug, while the expression of TNFRSF19 was associated with six drugs, suggesting their wide therapeutic effects. These results revealed that contribution of DEGs as a predictor of anticancer drug response. 


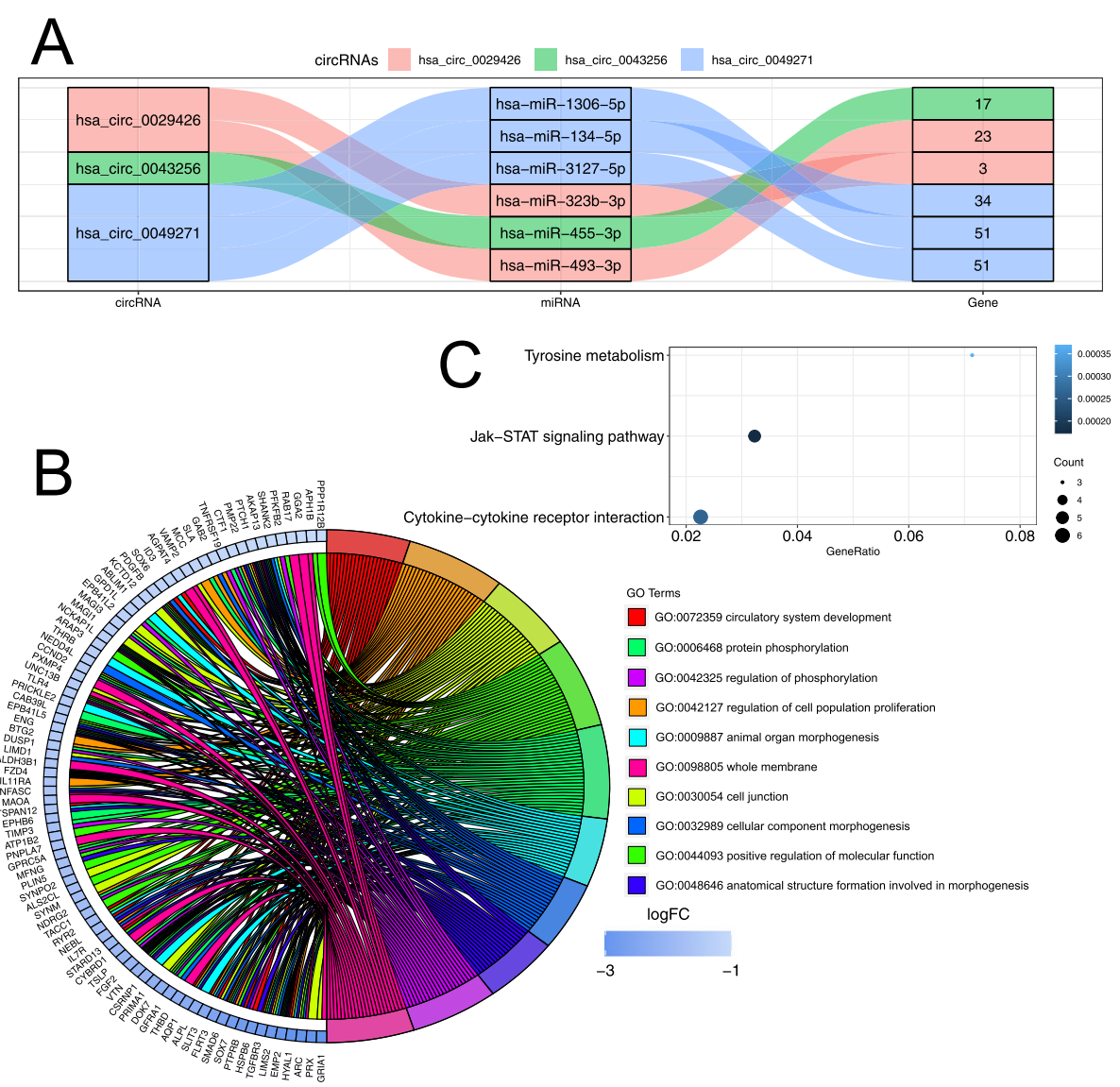

Fig. 3 The ceRNA network in LUAD. A Sankey diagram for the ceRNA network of hsa_circ_0049271, hsa_circ_0029426 and hsa_circ_0043256. B Distribution of DEGs regulated by hsa_circ_0049271 in LUAD for different GO-enriched functions. C Significant pathway enrichment of DEGs regulated by hsa_circ_0049271 in the ceRNA network

\section{Discussion}

Approximately 2.1 million new cases and 1.8 million deaths of lung cancer occur each year, making LUAD the cause of cancer incidence and mortality worldwide [34]. The early onset of LUAD is hard to screen, which leads to most of LUAD patients are usually found in the advanced stages of the disease. Therefore, Elucidating the molecular mechanisms of the tumorigenesis of LUAD is very important for identifying novel therapeutic targets and improving the clinical outcomes of LUAD patients. More and more studies have showed that circRNAs can serve as biomarkers for cancer diagnosis [35]. Compared to the traditional biomarkers, circRNAs are highly stable and conservative. Growing experimental evidence have proven that some circRNAs play important roles in the occurrence and development of LUAD acting as ceRNA [17, 36, 37]. The ceRNA hypothesis is a novel mechanism of RNA regulation by competing for shared miRNA response elements and provides important theoretical guidance for further understanding the tumorigenesis mechanism. However, the expression pattern of ceRNA in LUAD have not been thoroughly elucidated.

In our study, two microarray expression profile datasets from GEO dataset were used to identify DEcircRNAs in LUAD. Ten DEcircRNAs (5 upregulated and 5 downregulated) were significantly overlapped in two datasets, indicating important roles of circRNAs in LUAD. Moreover, hsa_circ_0000519 (circRNA-002178), hsa_circ_0072088 were identified, which were significantly upregulated in LUAD, and JunFeng Wang et al. revealed that circRNA-002178 could act as a miRNA sponge to promote PDL1/PD1 expression in LUAD [38], Liming Liang et al. confirmed that hsa_circ_0072088 were found differentially upregulated expression in LUAD with matched normal tissues by qRT-PCR with a $P$ value $<0.05$ [39]. However, circRNA-002178 was not in our ceRNA network based on our criteria (see methods). Because, we found that hsa-miR-34 regulated by circRNA-002178 was highly expressed in TCGALUAD tumor tissuesin our study and website tool (http://bioinfo.jialab-ucr.org/CancerMIRNome/) [40]. It was not consistent with previous result Wang et al. 

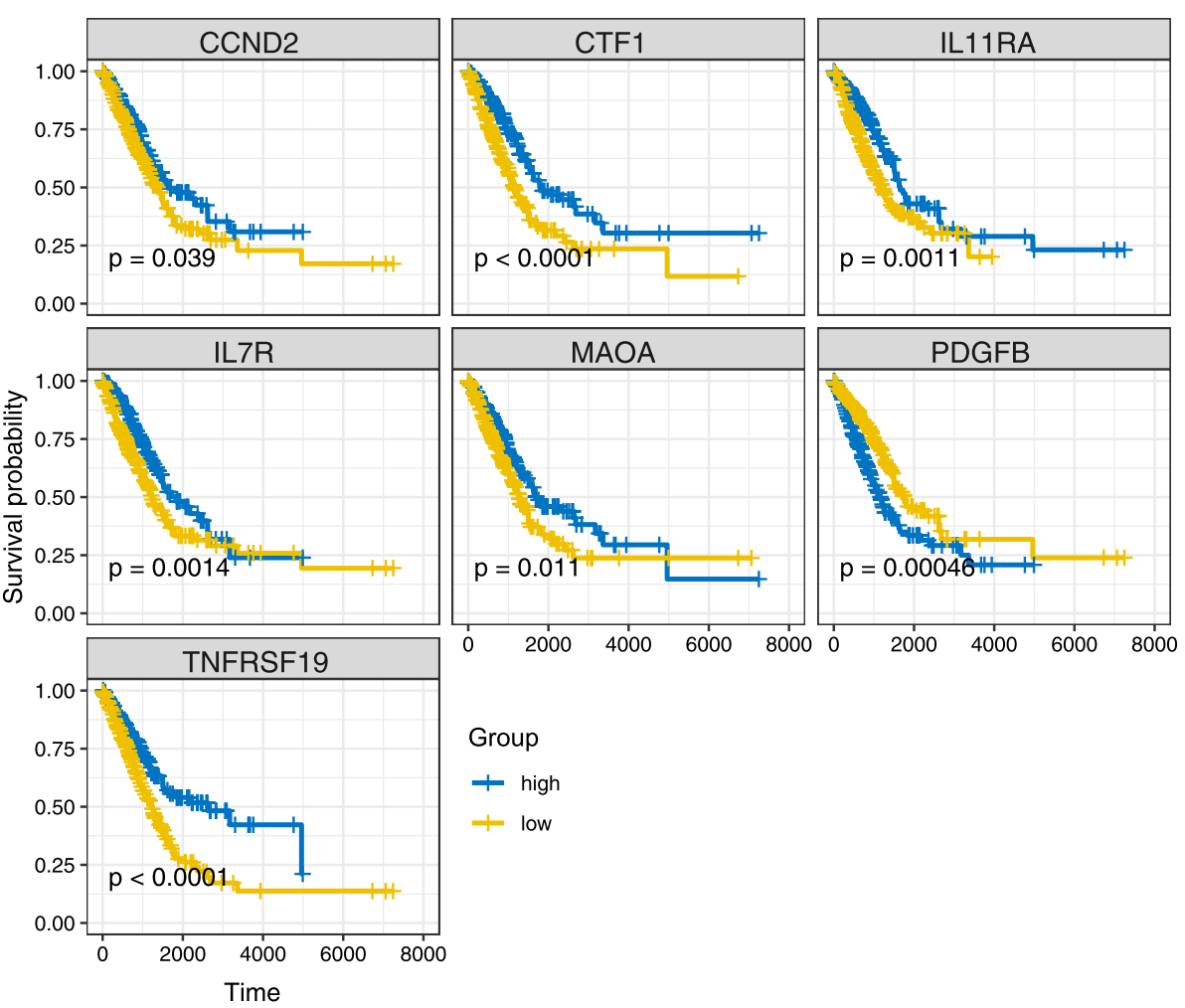

Group

+ high

+ low

Fig. 4 Kaplan-Meier survival curves of seven prognostic DEGs

reported that hsa-miR-34 significantly downregulated in LUAD cancer tissues. We chose the same miRNAs expression profiles from TCGA-LUAD dataset as Wang et al's. We analyzed the miRNA differential expression profiles of the 450 LUAD tissues and 45 non-tumor tissues. However, Wang et al's study did not provide the TCGA samples number and samples name of LUAD tissues and non-tumor tissues they used. Therefore, we guessed the inconsistent result due to different selection of tissue samples, and excluded circRNA-002178 ceRNA
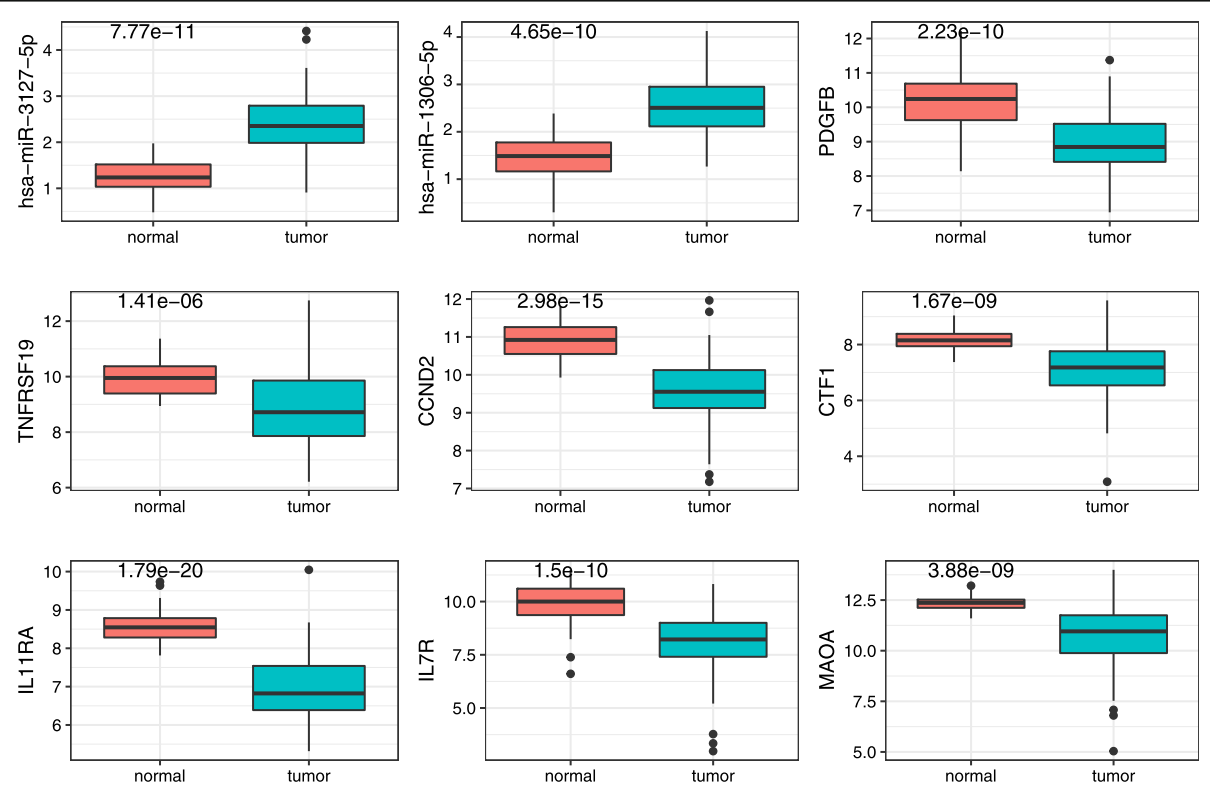

Fig. 5 Differentially expressed transcript regulated by hsa_circ_0049271 


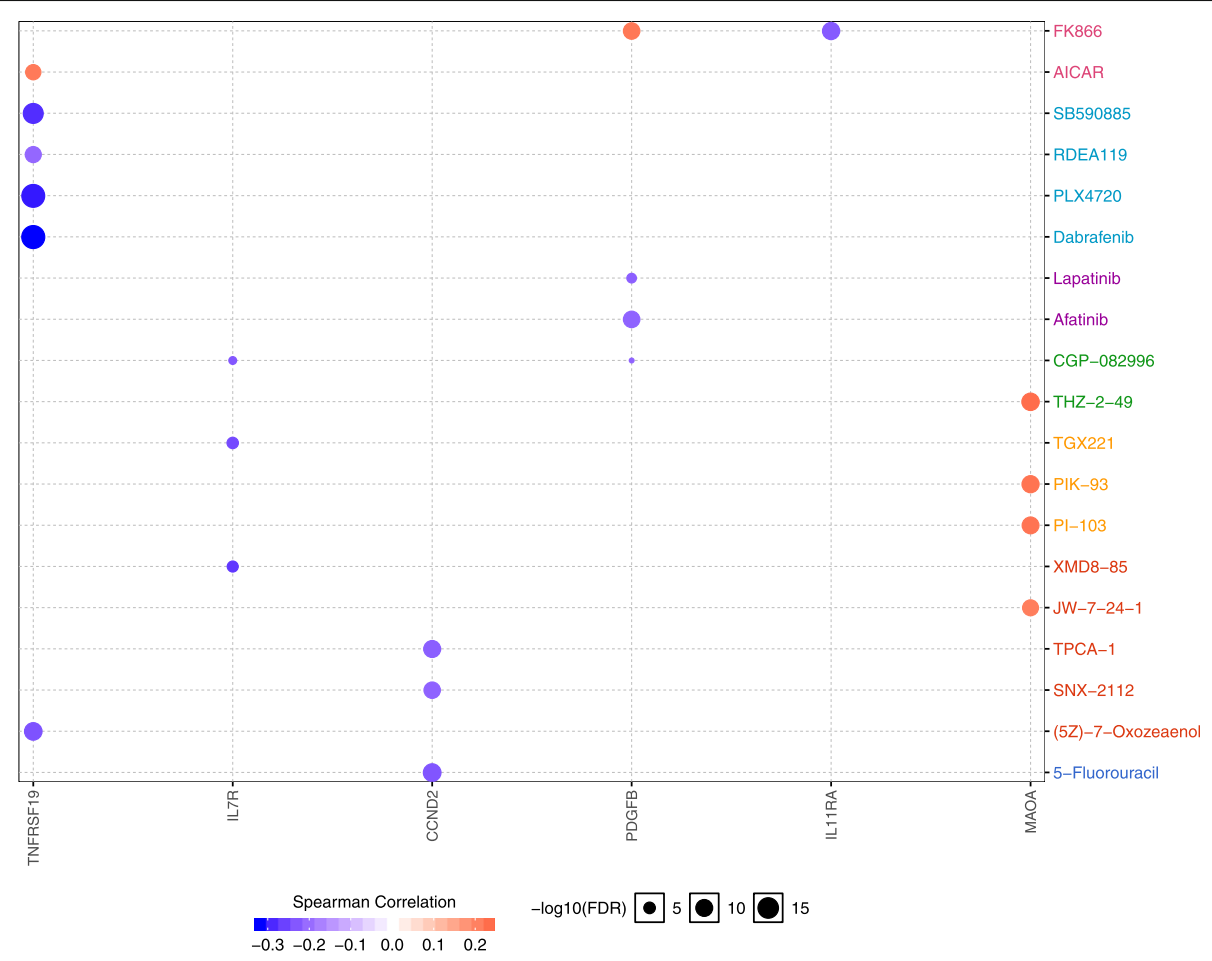

Fig. 6 Contribution of DEGs as a Predictor of Anticancer Drug Response

network for further analysis. In addition, hsa_circ 0043256 was significantly downregulated in LUAD. As prior study showed that hsa_circ_0043256 could inhibit cell proliferation and induced apoptosis in A549 and NCI-H460 cells [18]. These findings suggest that our bioinformatics method is valid for identifying potential function of circRNAs.

To investigate the roles of circRNAs in LUAD, we analyzed the expression profiles of their host genes in TCGA-LUAD samples. However, only very few genes were differential expression genes. Thereby, we hypothesis that circRNAs have more widely impact on transcripts via sponging miRNAs. Based on the ceRNA hypothesis, 10 DEcircRNAs, 147 DEmiRNAs and 4222 DEGs were selected to construct the circRNA-miRNAmRNA regulatory network, and we found that hsa_circ 0049271 interacted with most nodes. The GO enrichment analysis indicated that the function of 127 DEGs regulated by hsa_circ_0049271 could be involved in cell proliferation. Through KEGG pathway analysis, Cytokine-cytokine receptor interaction (map 04060), Jak-STAT signaling pathway (map 04630) and Tyrosine metabolism (map 00350) were detected, which are cancer-related pathways. The Jak-STAT signaling pathway is significantly involved in the development of solid tumors [41]. G Guney Eskiler et al. showed that IL-6 mRNA levels considerably increased $5.18 \pm 2.81$-fold in lung cancer, and the changes in the STAT3 and IL-6 expression levels may mediate STAT3 activation [42]. In addition, crizotinib reported by Anh et al. [43] and Hongmin $\mathrm{Lu}$ et al. [44] can promote apoptosis and inhibit cell proliferation of cells through JAK-STAT pathway in LUAD. Interestingly, the expression levels of four genes (PDGFB, CCND2, CTF1, IL7R) identified in the current study were strongly associated with STAT3 activation, clinical outcome of LUAD patients and drugs sensitivity across cancer cell lines in GDSC. All these above views indicated that the genes related to Jak-STAT signaling pathway reflects vital mechanisms of LUAD.

The expression levels of circRNA, miRNA and mRNA were comprehensively analyzed in TCGA-LUAD dataset. However, several limitations must be noted. First, DEcircRNAs identified in the present study were limited by sample size. Second, our findings need to be validated experimentally, which would be carried out in our following study.

\section{Conclusions}

In summary, a ceRNA network was successfully constructed, which including one circRNA, two miRNAs, and seven mRNAs. Seven mRNAs (PDGFB, TNFRSF19, CCND2, CTF1, IL11RA, IL7R and MAOA) were remarkably associated with the prognosis of LUAD patients. Among seven mRNA species, four genes (PDGFB, CCND2, CTF1, and IL7R) could be considered as drug targets in LUAD. 


\section{Abbreviations}

circRNAs: circular RNAs; LUAD: Lung adenocarcinoma; GO: Gene ontology; KEGG: Kyoto Encyclopedia of Genes and Genomes; ceRNA: Competing endogenous RNA; GEO: Gene Expression Omnibus; TCGA: The Cancer Genome Atlas

\section{Supplementary Information}

The online version contains supplementary material available at https://doi. org/10.1186/s12885-021-08462-8.

\section{Additional file 1. \\ Additional file 2. \\ Additional file 3. \\ Additional file 4. \\ Additional file 5. \\ Additional file 6. \\ Additional file 7. \\ Additional file 8. \\ Additional file 9. \\ Additional file 10. \\ Additional file 11. \\ Additional file 12. \\ Additional file 13.}

\section{Acknowledgements}

We would like to thank Jasonxu for constructive discussions.

\section{Authors' contributions}

The project was conceived and directed by Ling Zhang. Lei Gao collected data, and data analysis was performed by Lei Gao and Ling Zhang. Image processing was carried out by Lei Gao. The manuscript was written by Ling Zhang. All authors read and approved the final manuscript.

\section{Funding}

Not applicable.

\section{Availability of data and materials}

The authors declare that the data supporting the findings of this study are available within the article. The datasets generated and/or analysed during the current study are available in the GEO repository, https://www.ncbi.nlm. nih.gov/geo/. The RNA-seq data of raw and processed files have been deposited in NCBI Gene Expression Omnibus, accession number GSE101684, GSE101586 and GSE29249. All TCGA related data can be obtained from the TCGA database (https://tcga-data.nci.nih.gov/).

\section{Declarations}

Ethics approval and consent to participate

Not applicable.

\section{Consent for publication}

Not applicable.

\section{Competing interests}

The authors declare that they have no conflict of interest.

\section{Author details}

'Department of Respiratory and Critical Care Medicine, The Second Hospital of Anhui Medical University, 678 Furong Road, Economic And Technological Development Zone, Hefei 230601, Anhui Province, China. ${ }^{2}$ Department of Critical Care Medicine, The First Affiliated Hospital of Anhui Medical University, 218 Jixi Road, Shushan District, Hefei 230022, Anhui Province, China.
Received: 5 March 2021 Accepted: 8 June 2021

Published online: 23 July 2021

\section{References}

1. Siegel RL, Miller KD, Jemal A. Cancer statistics, 2020. CA Cancer J Clin. 2020; 70(1):7-30. https://doi.org/10.3322/caac.21590.

2. Qi L, Li Y, Qin Y, Shi G, Li T, Wang J, et al. An individualised signature for predicting response with concordant survival benefit for lung adenocarcinoma patients receiving platinum-based chemotherapy. $\mathrm{Br} \mathrm{J}$ Cancer. 2016;115(12):1513-9. https://doi.org/10.1038/bjc.2016.370.

3. Hirsch FR, Scagliotti GV, Mulshine JL, Kwon R, Curran WJ, Wu Y-L, et al. Lung cancer: current therapies and new targeted treatments. Lancet. 2017; 389(10066):299-311. https://doi.org/10.1016/S0140-6736(16)30958-8.

4. Vo JN, Cieslik M, Zhang Y, Shukla S, Xiao L, Zhang Y, et al. The Landscape of Circular RNA in Cancer. Cell. 2019;176:869-881.e13.

5. Rybak-Wolf A, Stottmeister C, Glažar P, Jens M, Pino N, Giusti S, et al. Circular RNAs in the mammalian brain are highly abundant, conserved, and dynamically expressed. Mol Cell. 2015;58(5):870-85. https://doi.org/10.1016/j. molcel.2015.03.027.

6. Hang D, Zhou J, Qin N, Zhou W, Ma H, Jin G, et al. A novel plasma circular RNA circFARSA is a potential biomarker for non-small cell lung cancer. Cancer Med. 2018;7(6):2783-91. https://doi.org/10.1002/cam4.1514.

7. Shang $Q$, Yang Z, Jia R, Ge S. The novel roles of circRNAs in human cancer. Mol Cancer. 2019;18(1):6. https://doi.org/10.1186/s12943-018-0934-6.

8. Kristensen LS, Hansen TB, Venø MT, Kjems J. Circular RNAs in cancer: opportunities and challenges in the field. Oncogene. 2018;37(5):555-65. https://doi.org/10.1038/onc.2017.361.

9. Holdt LM, Kohlmaier A, Teupser D. Molecular roles and function of circular RNAs in eukaryotic cells. Cell Mol Life Sci. 2018;75(6):1071-98. https://doi. org/10.1007/s00018-017-2688-5.

10. Li X, Yang L, Chen L-L. The biogenesis, functions, and challenges of circular RNAs. Mol Cell. 2018;71(3):428-42. https://doi.org/10.1016/j.molcel.2018.06. 034.

11. Zhong Z, Huang M, LV M, He Y, Duan C, Zhang L, et al. Circular RNA MYLK as a competing endogenous RNA promotes bladder cancer progression through modulating VEGFANEGFR2 signaling pathway. Cancer Lett. 2017; 403:305-17. https://doi.org/10.1016/j.canlet.2017.06.027.

12. Zeng K, Chen X, Xu M, Liu X, Hu X, Xu T, et al. CircHIPK3 promotes colorectal cancer growth and metastasis by sponging miR-7. Cell Death Dis. 2018;9(4):417. https://doi.org/10.1038/s41419-018-0454-8.

13. Zhang $X$, Wang S, Wang H, Cao J, Huang $X$, Chen Z, et al. Circular RNA circNRIP1 acts as a microRNA-149-5p sponge to promote gastric cancer progression via the AKT1/mTOR pathway. Mol Cancer. 2019;18(1):20. https:// doi.org/10.1186/s12943-018-0935-5.

14. Zeng K, He B, Yang BB, Xu T, Chen X, Xu M, et al. The pro-metastasis effect of circANKS1B in breast cancer. Mol Cancer. 2018;17(1):160. https://doi.org/1 0.1186/s12943-018-0914-X.

15. Ying $X$, Zhu J, Zhang Y. Circular RNA circ-TSPAN4 promotes lung adenocarcinoma metastasis by upregulating ZEB1 via sponging miR-665. Mol Genet Genomic Med. 2019;7:e991.

16. Du J, Zhang G, Qiu H, Yu H, Yuan W. The novel circular RNA circ-CAMK2A enhances lung adenocarcinoma metastasis by regulating the miR-615-5p/ fibronectin 1 pathway. Cell Mol Biol Lett. 2019;24(1):72. https://doi.org/10.11 86/s11658-019-0198-1.

17. Yao Y, Hua Q, Zhou Y. CircRNA has_circ_0006427 suppresses the progression of lung adenocarcinoma by regulating miR-6783-3p/DKK1 axis and inactivating Wnt/ $\beta$-catenin signaling pathway. Biochem Biophys Res Commun. 2019;508(1):37-45. https://doi.org/10.1016/j.bbrc.2018.11.079.

18. Wang $X$, Zhu $X$, Zhang H, Wei S, Chen Y, Chen Y, et al. Increased circular RNA hsa_circ_0012673 acts as a sponge of miR-22 to promote lung adenocarcinoma proliferation. Biochem Biophys Res Commun. 2018;496(4): 1069-75. https://doi.org/10.1016/j.bbrc.2018.01.126.

19. Hansen TB, Jensen TI, Clausen BH, Bramsen JB, Finsen B, Damgaard CK, et al. Natural RNA circles function as efficient microRNA sponges. Nature. 2013; 495(7441):384-8. https://doi.org/10.1038/nature11993.

20. Chen J, Song Y, Li M, Zhang Y, Lin T, Sun J, et al. Comprehensive analysis of ceRNA networks reveals prognostic IncRNAs related to immune infiltration in colorectal cancer. BMC Cancer. 2021;21(1):255. https://doi.org/10.1186/s12 885-021-07995-2. 
21. Zhao J, Li L, Wang Q, Han H, Zhan Q, Xu M. CircRNA expression profile in early-stage lung adenocarcinoma patients. Cell Physiol Biochem. 2017;44(6): 2138-46. https://doi.org/10.1159/000485953.

22. Chen T, Yang Z, Liu C, Wang L, Yang J, Chen L, et al. Circ 0078767 suppresses non-small-cell lung cancer by protecting RASSF1A expression via sponging miR-330-3p. Cell Prolif. 2019;52(2):e12548. https://doi.org/10.1111/ cpr.12548.

23. Ma L, Huang Y, Zhu W, Zhou S, Zhou J, Zeng F, et al. An integrated analysis of miRNA and mRNA expressions in non-small cell lung cancers. PLoS One. 2011;6(10):e26502. https://doi.org/10.1371/journal.pone.0026502.

24. Goldman MJ, Craft B, Hastie M, Repečka K, McDade F, Kamath A, et al. Visualizing and interpreting cancer genomics data via the Xena platform. Nat Biotechnol. 2020;38(6):675-8. https://doi.org/10.1038/s41587-020-0546-8.

25. Glažar P, Papavasileiou P, Rajewsky N. circBase: a database for circular RNAs. RNA. 2014;20(11):1666-70. https://doi.org/10.1261/rna.043687.113.

26. Griffiths-Jones S, Saini HK, van Dongen S, Enright AJ. miRBase: tools for microRNA genomics. Nucleic Acids Res. 2008;36(suppl_1):D154-8.

27. Ritchie ME, Phipson B, Wu D, Hu Y, Law CW, Shi W, et al. limma powers differential expression analyses for RNA-sequencing and microarray studies. Nucleic Acids Res. 2015;43:e47.

28. Li R, Qu H, Wang S, Wei J, Zhang L, Ma R, et al. GDCRNATools: an R/ Bioconductor package for integrative analysis of IncRNA, miRNA and mRNA data in GDC. Bioinformatics. 2018;34(14):2515-7. https://doi.org/10.1093/ bioinformatics/bty 124 .

29. Kanehisa M, Furumichi M, Sato Y, Ishiguro-Watanabe M, Tanabe M. KEGG: integrating viruses and cellular organisms. Nucleic Acids Res. 2020;49:D54551.

30. Subramanian A, Tamayo P, Mootha VK, Mukherjee S, Ebert BL, Gillette MA, et al. Gene set enrichment analysis: a knowledge-based approach for interpreting genome-wide expression profiles. Proc Natl Acad Sci U S A. 2005;102(43):15545-50. https://doi.org/10.1073/pnas.0506580102.

31. Liu C-J, Hu F-F, Xia M-X, Han L, Zhang Q, Guo A-Y. GSCALite: a web server for gene set cancer analysis. Bioinformatics. 2018;34(21):3771-2. https://doi. org/10.1093/bioinformatics/bty411.

32. Iorio F, Knijnenburg TA, Vis DJ, Bignell GR, Menden MP, Schubert M, et al. A landscape of Pharmacogenomic interactions in Cancer. Cell. 2016;166(3): 740-54. https://doi.org/10.1016/j.cell.2016.06.017.

33. Garnett MJ, Edelman EJ, Heidorn SJ, Greenman CD, Dastur A, Lau KW, et al. Systematic identification of genomic markers of drug sensitivity in cancer cells. Nature. 2012;483(7391):570-5. https://doi.org/10.1038/nature11005.

34. Bray F, Ferlay J, Soerjomataram I, Siegel RL, Torre LA, Jemal A. Global cancer statistics 2018: GLOBOCAN estimates of incidence and mortality worldwide for 36 cancers in 185 countries. CA Cancer J Clin. 2018;68(6):394-424. https://doi.org/10.3322/caac.21492.

35. Han B, Chao J, Yao H. Circular RNA and its mechanisms in disease: from the bench to the clinic. Pharmacol Ther. 2018;187:31-44. https://doi.org/10.101 6/j.pharmthera.2018.01.010.

36. Gao N, Ye B. Circ-SOX4 drives the tumorigenesis and development of lung adenocarcinoma via sponging miR-1270 and modulating PLAGL2 to activate WNT signaling pathway. Cancer Cell Int. 2020;20(1):2. https://doi. org/10.1186/s12935-019-1065-x.

37. Liang Z-Z, Guo C, Zou M-M, Meng P, Zhang T-T. circRNA-miRNA-mRNA regulatory network in human lung cancer: an update. Cancer Cell Int. 2020; 20:173.

38. Wang J, Zhao X, Wang Y, Ren F, Sun D, Yan Y, et al. circRNA-002178 act as a ceRNA to promote PDL1/PD1 expression in lung adenocarcinoma. Cell Death Dis. 2020;11:1-11.

39. Liang L, Zhang L, Zhang J, Bai S, Fu H. Identification of circRNA-miRNAmRNA networks for exploring the fundamental mechanism in lung adenocarcinoma. Onco Targets Ther. 2020;13:2945-55. https://doi.org/10.214 7/OTT.S235664.

40. Li R, Qu H, Wang S, Wang X, Cui Y, Yu L, et al. CancerMIRNome: a web server for interactive analysis and visualization of cancer miRNome data. bioRxiv. 2020; 2020.10.04.325670.

41. Yang X, Tang Z, Zhang P, Zhang L. Research advances of JAK/STAT signaling pathway in lung Cancer. Zhongguo Fei Ai Za Zhi. 2019;22(1):4551. https://doi.org/10.3779/j.issn.1009-3419.2019.01.09.

42. Eskiler GG, Bezdegumeli E, Ozman Z, Ozkan AD, Bilir C, Kucukakca BN, et al. IL-6 mediated JAK/STAT3 signaling pathway in cancer patients with cachexia. Bratisl Lek Listy. 2019;66(11):819-26. https://doi.org/10.4149/BLL 2 019_136.
43. Ahn HK, Jeon K, Yoo H, Han B, Lee SJ, Park H, et al. Successful treatment with crizotinib in mechanically ventilated patients with ALK positive nonsmall-cell lung cancer. J Thorac Oncol. 2013;8(2):250-3. https://doi.org/10.1 097/JTO.0b013e3182746772

44. Lu H, Wu S, Chen H, Huang Y, Qiu G, Liu L, et al. Crizotinib induces apoptosis of lung cancer cells through JAK-STAT pathway. Oncol Lett. 2018; 16(5):5992-6. https://doi.org/10.3892/ol.2018.9387.

\section{Publisher's Note}

Springer Nature remains neutral with regard to jurisdictional claims in published maps and institutional affiliations.
Ready to submit your research? Choose BMC and benefit from:

- fast, convenient online submission

- thorough peer review by experienced researchers in your field

- rapid publication on acceptance

- support for research data, including large and complex data types

- gold Open Access which fosters wider collaboration and increased citations

- maximum visibility for your research: over $100 \mathrm{M}$ website views per year

At BMC, research is always in progress.

Learn more biomedcentral.com/submissions 